\title{
EPJ Applied Metamaterials Special Issue on "Metamaterial-by-Design: Theory, Methods, and Applications to Communications and Sensing"
}

\author{
Guest Editors: Andrea Massa* and Giacomo Oliveri**
}

Received 30 August 2016 / Accepted 6 September 2016

In the recent years there has been an increasing interest in the possibility to engineer materials able to exhibit desired effective physical properties because of their potential applications in several areas. Indeed, the possibility to reduce the cost, enhance the features and flexibility, and obtain behaviors not achievable with standard media simply by combining (through suitably mixtures/ordered arrangements) common materials has gathered the interest of a vast community of engineers, scientists, chemists, and physics with strong interdisciplinary skills. The recent introduction of a wide class of new artificial materials (including, but not limited to, nano-materials, bio-materials, graphene and its composites, and metamaterials) has then further fostered the emergence of a completely new area of science and engineering, with important applications already demonstrated in academic and industrial scenarios. Such initial results in turns have motivated the development and refinement of many new design approaches, synthesis strategies, and fabrication technologies.

In this framework, "Metamaterials-by-Design" (MbD) has emerged as a powerful paradigm to address the above challenges. $M b D$ is an instance of the System-by-Design paradigm [1-4] defined in short as "How to deal with complexity". More specifically, $M b D$ considers the problem of designing artificial-material enhanced-devices from a completely new perspective, that is

The application-oriented synthesis of advanced systems comprising artificial materials whose constituent properties are driven by the device functional requirements.

This paradigm is based on the concept that any device comprising artificial materials must be designed (i) considering its functionality as the fundamental objective for the synthesis, and that (ii) the properties of the media employed in the device must be used as the fundamental degrees-of-freedom during the synthesis process.

\footnotetext{
*e-mail: andrea.massa@l2s.centralesupelec.fr

**e-mail: giacomo.oliveri@unitn.it
}

The Special Issue of the EPJ Applied Metamaterials, which is dedicated to the first edition of the "International Workshop on Metamaterial-by-Design: Theory, Methods, and Applications to Communications and Sensing" held in Paris (France) in December 3-4, 2015, is aimed at presenting an overview of the latest advances and emerging trends in the theory, methods, and the Communications and Sensing applications of the Material-by-Design paradigm, as well as at highlighting the current status and the envisaged frontiers in this research area. To this end, the Special Issue includes nine papers representing the state-of-the-art work being carried out in this famework by some of the top University research labs around the world.

More in detail, in terms of theoretical tools useful for MbD methodologies, Moccia et al. [5] address the synthesis and control of degenerate-band-edge effects in nonlocal metamaterials. The proposed concept, which is inspired by nonlocal-transformation-optics approaches, is based on the approximation of analytically-derived nonlocal constitutive "blueprints". Such a methodological approach enables to analyze and describe the physical mechanisms underlying the designed systems, thus providing an additional insight in the motivation and possible developments of such devices. The synthesis procedure is illustrated and validated on multilayered metamaterials featuring anisotropic constituents, and its potential application in slow light, solid-state lasers, quantum-cascade lasers, sensors, optical delay lines, traveling-wave tubes, distributed solid-state amplifiers, and switches is pointed out.

Moreover, a full-wave point-dipole-based scheme for accurate material homogenization of composites (i.e., a fundamental functional block in $\mathrm{MbD}$ synthesis procedures) is introduced in the paper by Naeem and Hao [6]. The method efficiently computes the interactions between the scatterers (even in inhomogeneous mixtures and aperiodic arrangements) by utilizing each inclusion as a basis function. Thanks to the capability to avoid the computational burden to discretize each inclusion scatterer (which is usually required in conventional homogenization approaches based on periodic boundary conditions), the proposed technique provides closed-form 
expressions for the mutual interaction between the scatterers. The proposed homogenization scheme is validated in the extraction of the permittivity profiles of finite size specimens.

Within the same framework, Moeini and Pedro [7] introduce a transfer matrix-based homogenization approach applicable to self-similar fractal structures. The proposed methodology enables to analytically compute the material parameters of a layered metamaterial comprising two different dielectrics distributed according to a fractal set. To this end, an innovative homogenization process is introduced which exploit the self-similarity properties of fractals. The obtained band dispersion diagrams and effective permittivities are numerically verified through full-wave simulations.

As regards guided wave $\mathrm{MbD}$ applications, a tri-band Branch-Line Coupler (BLC) based on a double-Lorentz (DL) transmission line (TL) metamaterial is presented in the paper by Mazeh et al. [8]. Thanks to the flexibility in the phase response characteristic of BLC, tri-band operation is achieved by using an even-odd mode analysis strategy. The introduced approach is validated in the design of a coupler with transmission coefficient equal to $3 \pm 0.5 \mathrm{~dB}$, return losses and isolations larger than $14 \mathrm{~dB}$, and a phase difference of $\pm 90 \pm 3.5^{\circ}$, and suitable for GSM-UMTS applications.

In the framework of $\mathrm{MbD}$ applications to field manipulation, Joshi et al. [9] introduce an innovative MbD synthesis of a dielectric superstrate which is capable of reorienting the electromagnetic waves emanating from a directive antenna in the azimuthal plane. The design procedure, based on Transformation Optics theory, enables to analytically derive a dielectric-only solution to the synthesis problem. The effectiveness of the proposed strategy is demonstrated by designing and numerically validating a dielectric superstrate able to completely reorient the electromagnetic waves of a classical patch antenna. Thanks to the introduced strategy, it is demonstrated that an antipodal radiation pattern can be obtained by compact low profile antennas combined with field manipulation devices comprising standard dielectric materials.

The paper by Herrojo et al. [10] presents the study, design, and full-wave simulation of miniaturized barcodes exploiting S-shaped split ring resonators (S-SRRs) through an innovative MbD-inspired technique. Thanks to their "S" shape, such resonators are shown to enable multiple controllable resonances and therefore to allow achieving spectral signature control by integration with coplanar waveguide (CPW) transmission lines. The effectiveness of the introduced geometry and design technique (in terms of RF performance and miniaturization) is validated in the design of a 10-bit barcode occupying $1 \mathrm{GHz}$ spectral bandwidth, centered at $2.5 \mathrm{GHz}$, and having a width of $9 \mathrm{~cm}^{2}$.

Ratni et al. [11] discuss the MbD-inspired synthesis and engineering of metasurfaces for antenna and sensing applications. More in detail, the possibility to steer the antenna radiation beam through phase modulated metasurfaces is firstly addressed by operating on its local inductance. Then, a sensing metasurface is designed by using a dark mode excitation mechanism based on the symmetry of the matching conditions, which allows to considerably relax the tolerances for the fabrication of such nanostructures at optical frequencies.
The introduced synthesis procedures are numerically (using full-wave finite element method-based commercial softwares HFSS from ANSYS) and experimentally validated.

Moreover, the paper by Rodríguez-Ulibarri et al. [12] is aimed at presenting two MbD-inspired solutions for the mitigation of the scan blindness in phased array antennas. The proposed strategies are based either on the introduction of portions of a bed of nails are in the radome to prevent the excitation of surface waves, or in the synthesis of a superstrate metasurface to achieve a wide angle impedance matching. Beyond the full-wave numerical simulations, a 100-element prototype phased array is used to validate the proposed approach also from the experimental viewpoint.

Finally, a comprehensive review on MbD-inspired methodologies and technologies for manipulating electromagnetic scattering by using metamaterials and metasurfaces is presented in the paper by Lu et al. [13]. Many approaches for controlling the field scattered by objects are presented, including various cloaking devices (e.g., invisibility cloaks, carpet cloaks, mantle cloaks, "skin" cloaks) exploiting different methodological approaches (transformation optics, gradient metamaterials, scattering cancellation, phase compensation, etc.), together with radar illusion devices, scattering controls with coding/programmable metasurfaces, and scattering reductions by multilayered structures. Future development of metamaterials on scattering manipulation are illustrated, as well.

To conclude this editorial, we would like to thank the EPJ Applied Metamaterials for the opportunity to organize such this Special Issue and to serve as Guest Editors. Moreover, we would like to thank all of the authors for their contributions and for their patience in the revision process. We hope that you will find this Special Issue on the subject of Metamaterial-byDesign interesting. The work presented in these papers shows that such a topic currently represents an extremely active and interdisciplinary research field with significant scientific and industrial potentialities.

\section{AndRea Massa, Guest Editor Giacomo Oliveri, Guest Editor}

Acknowledgements. This work has been partially supported by the TRIDENT Project (Convention de financement DIGITEO $\mathrm{N}^{\circ} 2015-1848 \mathrm{D}$ ) funded by DIGITEO (France), and it benefited from the networking activities carried out within the SIRENA project (2014-2017) funded by DIGITEO (France) under the "Call for Chairs 2014", and within the "Jean d'Alembert" Scholarship Program (2016-2017) funded by the IDEX Université Paris Saclay (France).

\section{References}

1. A. Massa, G. Oliveri, P. Rocca, and F. Viani, "System-byDesign: a new paradigm for handling design complexity", 8th European Conference on Antennas Propag. (EuCAP 2014), The Hague, The Netherlands, pp. 1180-1183, April, 2014.

2. G. Oliveri, L. Tenuti, E. Bekele, M. Carlin, A. Massa, An SbD-QCTO approach to the synthesis of isotropic metamaterial lenses, IEEE Antennas Wireless Propag. Lett. 13 (2014) $1783-1786$. 
3. G. Oliveri, F. Viani, N. Anselmi, A. Massa, Synthesis of multilayer WAIM coatings for planar phased arrays within the system-by-design framework, IEEE Trans. Antennas Propag. 63 (2015) 2482-2496.

4. G. Oliveri, D.H. Werner, A. Massa, Reconfigurable electromagnetics through metamaterials - A review, Proc. IEEE 103 (Jul. 2015) 1034-1056.

5. M. Moccia, G. Castaldi, V. Galdi, Degenerate-band-edge engineering inspired by nonlocal transformation optics, EPJ Appl. Metamat. 3 (2016) 1.

6. M. Naeem, Y. Hao, Homogenization of composites using full-wave point-dipole model, EPJ Appl. Metamat. 3 (2016) 6.

7. S. Moeini, J.C. Pedro, Extraction of material parameters in 1-D fractal metamaterial, EPJ Appl. Metamat. 3 (2016) 7.

8. F. Mazeh, H. Ayad, M. Fadlallah, K. Joumaa, J. Jomaah, F. Ndagijimana, Even-odd mode of a double-Lorentz metamaterial and its application to a tri-band branch-line coupler, EPJ Appl. Metamat. 3 (2016) 8.
9. C. Joshi, A.C. Lepage, X. Begaud, A dielectric-only superstrate inspired from transformation optics for complete reorientation of electromagnetic waves in azimuthal plane, EPJ Appl. Metamat. 3 (2016) 5.

10. C. Herrojo, J. Naqui, F. Paredes, F. Martín, Spectral signature barcodes based on S-shaped Split Ring Resonators (S-64 SRRs), EPJ Appl. Metamat. 3 (2016) 1.

11. B. Ratni, E. Bochkova, G.-P. Piau, A. de Lustrac, A. Lupu, S.N. Burokur, Design and engineering of metasurfaces for highdirectivity antenna and sensing applications, EPJ Appl. Metamat. 3 (2016) 1-10.

12. P. Rodríguez-Ulibarri, T. Crépin, C. Martel, F. Boust, F. Falcone, C. Loecker, K. Herbertz, T. Bertuch, T. Dousset, J.-P. Martinaud, S. Maci, J.A. Marcotegui, M. Beruete, Experimental demonstration of metamaterials application for mitigating scan blindness in phased array antennas, EPJ Appl. Metamat. 3 (2016) 9.

13. C. Lu, Z.L. Mei, W.X. Tang, T.J. Cui, Manipulating scattering features by metamaterials, EPJ Appl. Metamat. 3 (2016) 3.

Cite this article as: Massa A \& Oliveri G: EPJ Applied Metamaterials Special Issue on "Metamaterial-by-Design: Theory, Methods, and Applications to Communications and Sensing”. EPJ Appl. Metamat. 2016, 3, E1. 